\title{
The Use of Methylphenidate in the Treatment of ADHD: What is the Mechanism for Treating Working Memory Deficits?
}

\author{
Nidhila Masha $^{1 *}$ and Kathryn Dickerson ${ }^{1}$
}

Methylphenidate (MP), a drug which blocks dopamine reuptake, is most commonly used to treat Attention Deficit Hyperactivity Disorder (ADHD), a disorder associated with a disrupted dopamine system in the brain. Though generally effective at treating common ADHD symptomology (e.g. hyperactivity and impulsivity), MP is less effective at treating working memory deficits, which are also associated with ADHD. Within working memory, MP is more effective at treating deficits in the visuospatial component than it is in treating deficits in the audio-verbal component. This review aims to draw attention to the need for further research on the mechanisms through which MP alleviates working memory deficits. Determining these mechanisms may allow researchers to start developing ways to increase the efficacy of this drug at treating problems with auditory-verbal working memory.

\section{INTRODUCTION}

Attention Deficit Hyperactivity Disorder (ADHD), which affects somewhere between $1-20 \%$ of school age children, is characterized by symptoms of inattention, hyperactivity and impulsivity (Polanczyk et al., 2007, DSM-IV). All three of these symptoms have been linked to abnormalities in the executive, frontal lobe systems of the brain (Lazar and Frank, 1998). Furthermore, children with ADHD are also at greater risk of presenting with other deficits in executive function including motivation, planning, and working memory (Edmund, 2003; Willcutt et al., 2005). This paper focuses specifically on the working memory deficits associated with ADHD, as prior studies have demonstrated that Methylphenidate (MP), the drug most commonly used to treat ADHD symptomology, is less effective at treating working memory deficits than it is at addressing the core symptoms of ADHD such as hyperactivity and impulsivity (Greenhill 2006; Bedard and Tannock, 2008). For example, while MP does successfully alleviate some working memory deficits associated with ADHD, its efficacy varies across the different modalities of working memory (Bedard and Tannock, 2008). In order to determine the cause of this variation, further research must be conducted on the mechanisms through which MP alleviates working memory deficits, which can significantly impact the quality of life of individuals with ADHD.

${ }^{1}$ Duke University, 308 Research Drive, LSRC, PO Box 90999 Durham, NC 27708

*To whom correspondence should be addressed: masha.nila@gmail.com

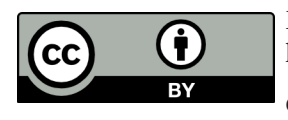

Except where otherwise noted, this work is licensed under https://creativecommons.org/licenses/by/4.0

doi:10.22186/jyi.37.5.39-42

\section{ADHD AND WORKING MEMORY}

Deficits in working memory are common in patients with ADHD (Barkley, 1997; Edmund, 2003; Willcutt et al., 2005). One theory suggests that these deficits may occur because working memory is modulated through dopaminergic systems (Goldman-Rakic et al., 2003), and ADHD is associated with abnormalities in the dopamine-reward pathway. Studies conducted on monkeys have shown that working memory deficits can be induced through administration of dopamine antagonists and that these induced deficits can be discontinued through subsequent administration of dopamine agonists (D'Esposito and Postle, 2015). Indeed, individuals with ADHD have been found to have fewer dopamine receptors and transporters available in the midbrain, where the majority of dopamine in the brain is produced, and in the nucleus accumbens, a structure that receives significant projections from the midbrain and is integral to the dopamine-reward pathway (Volkow et al., 2009).

According to the widely accepted Baddeley model (Baddeley, 1974), there are three components to working memory: the visuospatial circuit which manages visual information, the verbal circuit which manages auditory and phonological information, and the central executive, which controls engagement of the other two circuits and manages processes such as multitasking. Though little research has been done to investigate the impact of ADHD on central executive working memory, children with ADHD have been shown to exhibit greater deficits in visuospatial working memory than in auditory-verbal working memory (Martinussen et al., 2005). As a result, most studies analyzing the efficacy of different ADHD medications on working memory test the medications' effect on visuospatial working memory alone (Tannock et al., 1995; Bedard and Tannock, 2008). However, while deficits in auditory-verbal working memory are less severe than their visuospatial counterparts, such deficits are still common 
and serve as detriments to quality of life. Consequently, there is currently a need for more studies examining the efficacy of common treatments like MP on non-visuospatial components of working memory.

\section{METHYLPHENIDATE AS A TREATMENT FOR ADHD}

MP is the active component of the majority of drugs used to treat ADHD. It hinders the reuptake of dopamine, allowing for an accumulation of naturally released dopamine in the brain (Sonders et al., 1997). Since ADHD symptomology is believed to be caused, at least in part, by a shortage of dopamine transporters and receptors, this suggests that the accumulation of dopamine caused by MP would alleviate symptoms of ADHD. Studies investigating the efficacy of MP as a treatment for ADHD have confirmed that it successfully increases dopamine concentrations in the brain; patients who had been given MP were significantly more likely to report, or have guardians report, a remission of core ADHD symptoms than patients who were administered a placebo (Greenhill 2006).

Effects of Methylphenidate on different components of working memory

Methylphenidate has been shown to successfully alleviate some working memory deficits associated with ADHD (Tannock et al., 1995; Tannock et al., 1995; Bedard and Tannock, 2008). However, it achieves varying success across the different modalities of working memory. While little research has been done on MP's effect on central-executive working memory, several studies have tested its effect on visuospatial and auditory-verbal working memory (Tannock et al., 1995; Tannock et al., 1995; Bedard and Tannock, 2008). These studies have shown that MP is more successful in increasing visuospatial working memory than it is at increasing auditory-verbal working memory (Bedard and Tannock 2008). While this variation in efficacy of MP across different modalities of working memory is most pronounced in children with ADHD and co-morbid anxiety, it is also present in children who have ADHD but do not present with co-morbidities (Bedard and Tannock, 2008). Granted, there is an ongoing dispute about whether or not MP truly does have sub-optimal impacts on populations with co-morbid anxiety, with different studies providing conflicting evidence (Pliszka, 1989; Buitelaar et al., 1995; Tannock et al., 1995; Diamond et al., 1999). However, studies which demonstrated that MP is less effective at reducing behavioral symptoms of ADHD in individuals with co-morbid anxiety (Pliszka, 1989; Buitelaar et al., 1995; Tannock et al., 1995) measured behavioral symptoms immediately after or during a four-week MP administration trial. By contrast, the study which showed no significant difference in symptom reduction between individuals with and without comorbid anxiety (Diamond et al., 1999) measured behavioral symptoms four months after the MP administration trials. The implication suggests that, at least in the short-term, MP does have sub-optimal impacts on the symptomology of individuals with both ADHD and co-morbid anxiety. Since nearly $25 \%$ of children with ADHD also suffer from

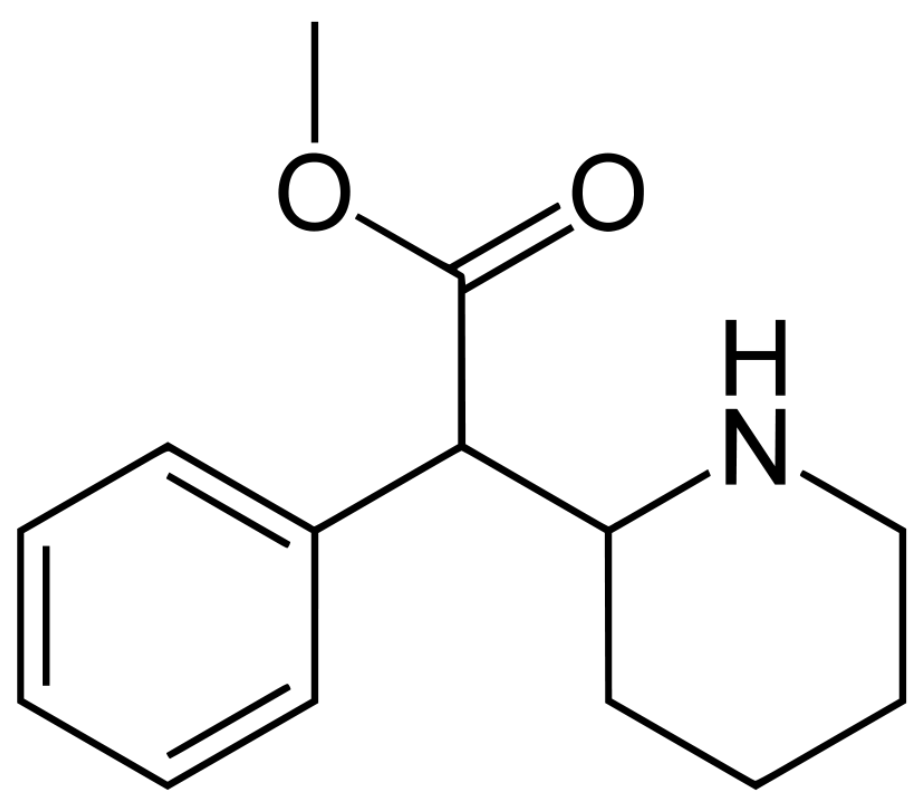

Figure 1. Molecular structure of Methylphenidate.

an anxiety disorder (Anderson et al., 1987), there is a significant need for research that explores and works towards amending MP's shortcomings in this specific clinical population. It is still unknown why MP is relatively ineffective at alleviating deficits in auditory verbal working memory, and why this inefficacy is more pronounced for ADHD children with co-morbid anxiety. The first step in solving this problem may be to investigate the mechanism through which MP alleviates working memory.

Need for further research on mechanism through which MP alleviates working memory

While several studies have shown that administration of MP alleviates ADHD-related deficits in working memory (Tannock et al., 1995; Bedard and Tannock, 2008), the mechanism through which the drug alleviates such deficits is unclear. It was initially assumed that because both core and non-core ADHD symptomology can be traced back to the dopamine-reward pathway, and because MP increases dopamine concentrations in the brain, MP achieved all its alleviatory effects - including that on working memory - by compensating for a shortage of dopamine transporters/receptors was the mechanism. However, a recent study demonstrated that MP-related dopamine increases have no effect on hyperactivity, though administration of MP has been shown to cause improvement in hyperactivity symptoms (Volkow et al., 2011). It follows that increasing dopamine signaling is not necessarily the mechanism through which MP exercises all its alleviatory effects. No study has yet proven or disproven the hypothesis that increasing dopamine signaling is the mechanism through which MP alleviates working memory deficits.

An Alternative Mechanism: MP's effect on working memory Methylphenidate's effect on working memory may be explained through alternate mechanisms. For example, MP may increase 
working memory by causing vasodilation of arteries which supply blood to the regions of the brain associated with working memory. Methylphenidate administration has been shown to increase blood flow to the dorsolateral prefrontal cortex, posterior parietal cortex and right cerebellum; these increases correspond to improvements in working memory (Mehta et al., 2000). However, it is still unclear how and why MP targets vasculature supplying these regions of the brain while sparing others.

\section{DISCUSSION}

While it is possible that MP improves working memory solely through increasing dopamine concentrations, as is currently believed, researchers have yet to categorically confirm or disprove this theory. Since it is also possible that MP improves working memory through multiple mechanisms, alternate ways through which MP might improve working memory should be investigated to assess whether MP-related increases in dopamine concentrations are correlated with improvements in working memory. One promising avenue of investigation includes exploring the specialized way in which MP seems to affect cerebral vasculature.

Such research on the mechanism through which MP alleviates working memory deficits could shed light on why MP is relatively unsuccessful at treating auditory-verbal working memory deficits, especially in children who suffer from both ADHD and co-morbid anxiety. A better understanding of why MP fails to adequately alleviate deficits in auditory-verbal working memory could inform studies aiming to improve its efficacy in this field or develop treatments capable of effectively addressing such deficits. Auditory-verbal working memory affects sentence processing as well as speed and accuracy of syntax comprehension (Caplan and Waters, 1999). Deficits in this modality of working memory could thus impact development of reading and writing skills. Developing treatments capable of combating deficits in this modality of working memory could thus greatly improve the quality of life of children with ADHD, especially those who also suffer from comorbid anxiety.

\section{ACKNOWLEDGMENTS}

Nidhila Masha would like to thank her mentor, Kathryn Dickerson, for her guidance, support and constructive suggestions.

\section{REFERENCES}

Anderson, J. C., Williams, S., McGee, R., Silva, P. A. (1987). DSM-III Disorders in Preadolescent Children. Archives of General Psychiatry, 44(1), 69-76, available: 10.1001/archpsyc.1987.01800130081010

Barkley, R. A. (1997). Behavioral inhibition, sustained attention, and executive functions: Constructing a unifying theory of ADHD. Psychological Bulletin, 121(1), 65-94, available: 10.1037/0033-2909.121.1.65.

Baddeley, A. D. (1992) Working memory. Science, 255(5044), 556-559, available: $10.1126 /$ science. 1736359 .

Bedard, A-C., Tannock, R. (2007). Anxiety, Methylphenidate Response, and Working Memory in Children With ADHD. Journal of Attention Disorders, 11(5), 546-557, available: 10.1177/1087054707311213.

Buitelaar, J., Van der Gaag, R., Swaab-Barneveld, H. S., Kuiper, M. (1995). Prediction of clinical response to methylphenidate in children with attentiondeficit hyperactivity disorder. Journal of the American Academy of Child
\& Adolescent Psychiatry, 34(8), 1025-1032, available: 10.1097/00004583199508000-00012.

Caplan, D. and Waters, G. S. (1999), Verbal Working Memory and Sentence Comprehension. Behavioral Brain Sci, 22(1), 77-94, available: 10.1017/ S0140525X99001788.

D'Esposito, M., Postle, B. (2014), The Cognitive Neuroscience of Working Memory. Annual Review Psychology, 66, 115-142, available: 10.1146/ annurev-psych-010814-015031.

Diamond, I., Tannock, R., Schachar, R. (1999), Response to methylphenidate in children with ADHD and comorbid anxiety. Journal of the American Academy of Child \& Adolescent Psychiatry, 38(4), 402-409, available: 10.1097/00004583-199904000-00012.

Edmund, J. S. S-B. (2003). The dual pathway model of AD/HD: an elaboration of neuro-developmental characteristics. Neuroscience and Biobehavioral Reviews, 27(7), 593-604, available: 10.1016/j.neubiorev.2003.08.005.

Gilliam, M., Stockman, M., Malek, M., Sharp, W., Greenstein, D., Lalonde, F., Clasen, L., Giedd, J., Rapoport, J., Shaw, P. (2011). Developmental trajectories of the corpus callosum in attention-deficit/hyperactivity disorder. Society of Biological Psychiatry. 69(9), 839-846, available: 10.1016/j.biopsych.2010.11.024.

Goldman-Rakic, P., Castner, S. A., Svensson, T. H., Siever, L. J., and Williams, G. V. (2004). Targeting the dopamine D1 receptor in schizophrenia: Insights for cognitive dysfunction. Psychopharmacology, 174(1), 3-16, available: $10.1007 / \mathrm{s} 00213-004-1793-y$.

Greenhill, L. L., Kollins, S., Abikoff, H., McCracken, J., Riddle, M. A., Swanson, J., McGough, J., Wigal, S., Wigal, T., Vitiello, B., Skrobala, A., Posner, K., Ghuman, J., Cunningham, C., Davies, M., Chuang, S., and Cooper, T. (2006). Efficacy and safety of immediate-release methylphenidate treatment for preschoolers with ADHD. Journal of the American Academy of Child \& Adolescent Psychiatry, 45(11), 1284-1293, available: 10.1097/01. chi.0000235077.32661.61.

Hynd, G. W., Hern, K., Novey, M. D., Eliopulos, D., Marshall, R., Gonzalez, J. J., and Voeller, K. K. (1993). Attention Deficit-Hyperactivity Disorder and Asymmetry. Journal of Child Neurology, 24, 141-146, available: 10.1177/088307389300800409.

Jensen, P. S., Hinshaw, S. P., Kraemer, H. C. (2000). ADHD Comorbidity Findings From the MTA Study: Comparing Comorbid Subgroups. Journal of the Academy of Child \& Adolescent Psychiatry, 40(2), 147-158, available: 10. 1177/088307389300800409.

Lazar, J. W. and Frank, Y. (1998). Frontal Systems Dysfunction in Children with Attention-Deficit Hyperactivity Disorder and Learning Disabilities. The Journal of Neuropsychiatry and Clinical Neurosciences, 10, 160-167.

Lou, H., Rosa, P., Pryds, O., Karrebaek, H., Lunding, J., Cumming, P., Gjedde, A (2004). ADHD: Increased dopamine receptor availability linked to attention deficit and low neonatal cerebral blood flow. Developmental Medicine \& Child Neurology, 46(3), 179-183, available: 10.1111/j.1469-8749.2004. tb00469.x.

Martinussen, R., Hayden, J., Hogg-Johnson, S., Tannock, R. (2005). A MetaAnalysis of Working Memory Impairments in Children With AttentionDeficit/Hyperactivity Disorder. Journal of the Academy of Child \& Adolescent Psychiatry, 44(4), 377-384, available: 10.1097/01.chi.0000153228.72591.73.

Mehta, M. A., Owen, A. M., Sahakian, B. J., Mavaddat, N., Pickard, J. D., Robbins, T. W. (2000). Methylphenidate Enhances Working Memory by Modulating Discrete Frontal and Parietal Lobe Regions in the Human Brain. The Journal of Neuroscience, 20(6), 1-6, available: 10.1523/JNEUROSCI.2006-j0004.2000.

Pliszka, S. (1989), Effect of Anxiety on Cognition, Behavior, and Stimulant Response in ADHD. Journal of the Academy of Child \& Adolescent Psychiatry, 28(6), 882-88, available: 10.1097/00004583-198911000-00012.

Polanczyk, G., Silva de Lima, M., Horta, B. L., Biederman, J., Rohde, L. A. (2007). The worldwide prevalence of ADHD: a systematic review and metaregression analysis. The American Journal of Psychiatry, 164(6), 942948, available: 10.1176/ajp.2007.164.6.942.

Sonders, M. S., Zhu, S-J, Zahniser, N. R., Kavanaugh, M. P., Amara, S. G. (1997). Multiple Ionic Conductances of the Human Dopamine Transporter: The Actions of Dopamine and Psychostimulants. The Journal of Neuroscience, 17(3), 
960-974, available: https://doi.org/10.1523/JNEUROSCI.17-03-00960.1997.

Tannock, R., Ickowicz, A., Schachar, R. (1995). Differential Effects of Methylphenidate on Working Memory in ADHD Children with and without Comorbid Anxiety. Journal of the Academy of Child \& Adolescent Psychiatry, 34(7), 886-896, available: 10.1097/00004583-199507000-00012.

Thapar, A., Holmes, J., Poulton, K., Harrington, R. (1999). Genetic basis of attention deficit and hyperactivity. British Journal of Psychiatry, 174(2), 105111, available: $10.1192 /$ bjp.174.2.105.

Valera, E. M., Faraone, S. V., Murray, K. E., Seidman, L. J. (2006). MetaAnalysis of Structural Imaging Findings in Attention-Deficit/Hyperactivity Disorder. Society of Biological Psychiatry, 61(12), 1361-1369, available: 10.1016/j.biopsych.2006.06.011.

Volkow, N. D., Wang, G., Fowler, J. S., Logan, J., Gerasimov, M., Maynard, L., Ding, Y., Gatley, S. J., Gifford, A., Franceschi, D. (2001) Therapeutic doses of oral methylphenidate significantly increase extracellular dopamine in the human brain. The Journal of Neuroscience, 21(2), 1-5, available: 10.1523/ JNEUROSCI.21-02-j0001.2001.

Volkow, N. D., Wang, G. J., Kollins, S. H., Wigal, T. L., Newcorn, J. H., Telang, F., Fowler, J. S., Zhu, W., Logan, J., Ma, Y., Pradhan, K., Wong, C., Swanson, J. M. (2009) Evaluating dopamine reward pathway in ADHD: clinical implications. The Journal of American Medical Association, 302(10), 10841091, available: 10.1001/jama.2009.1308.

Volkow, N. D., Wang, G-J., Newcorn, J. H., Kollins, S. H., Wigal, T. L., Telang, F., Fowler, J. S., Goldstein, R. Z., Klein, N., Logan, J., Wong, C., and Swanson, J. M. (2011). Motivation deficit in ADHD is associated with dysfunction of the dopamine reward pathway. Molecular Psychiatry, 16(11), 1147-1154, available: $10.1038 / \mathrm{mp} .2010 .97$.

Volkow, N. D., Wang, G-J., Tomasi, D., Kollins, S. H., Wigal, T. L., Newcorn, J. H., Telang, F. W., Fowler, J. S., Logan, J., Wong, C. T., and Swanson, J. M. (2011). Methylphenidate-Elicited Dopamine Increases in Ventral Striatum Are Associated with Long-Term Symptom Improvement in Adults with Attention Deficit Hyperactivity Disorder. The Journal of Neuroscience, 32(3), 841-849, available: 10.1523/JNEUROSCI.4461-11.2012.

Willcutt, E. G., Doyle, A. E., Nigg, J. T., Faraone, S. V., Pennington, B. F. (2005). Validity of the Executive Function Theory of Attention- Deficit/Hyperactivity Disorder: A Meta-Analytic Review. Society of Biological Psychiatry, 57(11), 1336-1346, available: 10.1016/j.biopsych.2005.02.006.

Wise, R.A. (2002). Brain reward circuitry: insights from unsensed incentives. Neuron, 36(2), 229-240, available: 10.1016/S0896-6273(02)00965-0.

American Psychiatric Association. (2000). Diagnostic and Statistical Manual of Mental Disorders (4thed., text rev.). Washington, DC: American Psychiatric Association. 